\title{
Recommendations regarding the Internet, its Influencers and its Users
}

Much of what has been said about cultural decision makers and influencers applies to the decision makers and influencers of the online world as well. In fact, online communication is part of the process of the construction of meaning that we have described above as culture. We address the Internet here separately and not among the recommendations to cultural decision makers and influencers in general because to a large extent the Internet is responsible for the explosion of Jew-hatred in the last decades.

The Internet provides antisemites of all colors with a means to spread their agitation unhindered both in the form of explicit hate speech and in implicit manifestations coded in indirect speech acts. A recent long-term study by Monika Schwarz-Friesel exposes the Internet as "the primary multiplier and locus for the transmission of manifestations of antisemitism" and points to a more than alarming development: "Expressions of anti-Semitic sentiment have increased significantly in the digital age." ${ }^{1}$ The main results of the long-term study are:

- This increase is accompanied by a qualitative radicalization and intensification of expressions of antisemitism.

- Consequently, antisemitism's scope for expression as well as the visibility of antisemitic sentiments have grown enormously online.

- The epoch-spanning reiteration of Judeophobic stereotypes and conspiracy phantasies is revealed in thousands of texts every day in the Internet.

- Classical hostility towards Jews remains the primary conceptual basis for present-day hatred of Jews; 54.02 percent (mean value) of all expressions of antisemitism display classical stereotypes.

- Muslim antisemitism is also marked by classical stereotypes of hostility towards Jews.

1 M. Schwarz-Friesel, Antisemitism 2.0 and the Cyberculture of Hate: Hostility towards Jews as a cultural constant and collective emotional value in the digital age (short version), published online in 2018 (https://www.linguistik.tu-berlin.de/fileadmin/fg72/Antisemitism_2.0_short_version_ final2.pdf), 3. A more detailed German version of the results of the DLG-funded long-term study "Antisemitismus im www" ["Antisemitism in the World Wide Web"] can be found on the website of the Technische Universität Berlin (https://www.linguistik.tu-berlin.de/fileadmin/fg72/Anti semitismus_2-0_Lang.pdf).

Ә OpenAccess. ( ) 2019, Armin Lange, Kerstin Mayerhofer, Dina Porat, Lawrence H. Schiffman, published by De Gruyter. (cc) BY-NC-ND This work is licensed under the Creative Commons Attribution-NonCommercialNoDerivatives 4.0 License https://doi.org/10.1515/9783110618594-029 
- With a mean value of 33.35 percent, Israel-centered antisemitism is a prevalent manifestation of hatred towards Jews, which is, however, conceptually and affectively determined by classical hatred of Jews.

- The "Israelization of antisemitic semantics" also manifests itself in issues entirely unrelated to the Middle East conflict.

- Hatred of Jews projected on to Israel by means of classical stereotypes perpetuates the tradition of radical and eliminatory hostility towards Jews, thus increasingly legitimizing antisemitism in civil society in the absence of counter-measures in the judiciary or politics.

- Despite differing political and ideological beliefs, expressions of antisemitism, regardless of where they originate, display a pronounced uniformity and homogeneity in their encoding of stereotypes and in their argumentation.

- The quotidian communication processes of non-extremist everyday users of social media are responsible for the dissemination and normalization of antisemitic attitudes; hatred of Jews is revealed as a phenomenon manifest in society as a whole and as an omnipresent aspect of cyberculture.

- At the same time, intensive defensive and relativization strategies are an integral element of antisemitic discourse.

- A very pronounced emotional dimension can be observed: Antisemitic pseudo-argumentation displays its own affect logic.

- Hatred of Jews reveals itself in the multimodal encoding on the web as a constant and collective emotional value of cultural memory.

In addition to the communication of antisemitism online, the Internet represents a tool for the marketing of antisemitic contents in printed (e.g., books), visual (e.g., DVDs) and oral forms (e.g., music-CDs). The latter problem is not restricted to radical right wing book dealers and music labels. Amazon is the largest Internet retailer. It markets both explicit and implicit manifestations of antisemitism. Examples include the promotion of a book by Joseph Goebbels and the Nazi cartoonist Mjölnir entitled Isidor that is a collection of typical Nazi antisemitic propaganda. In response to a letter of protest, Amazon.de answered that the company markets products with whose contents it might disagree but whose contents would be regarded as "worthy of discussion."2

When the World Wide Web was created, it was intended to be a platform for the free and uncensored exchange of ideas in order to make the world a better and more democratic place. It was and is abused, however, to spread hate

2 Email correspondence from Amazon representative, sent April 26 2018. 
and to incite persecution. While the example of child pornography shows that certain contents can be successfully suppressed and marginalized online, it also shows that beyond restricting contents to darknets, no form of hate speech or crime can be removed totally from the Internet. Furthermore, implicit manifestations of antisemitism online would remain mostly untouched from a prohibitive approach despite the fact that due to their subtlety they are much more poisonous to the mind than vulgar texts of extremists.

Everyday antisemitism is much more dangerous than Jew-hatred stemming from extremists since the multipliers and promotors on the mainstream levels of the Web enhance the normalization and social acceptability of antisemitism. Merely deleting antisemitic posts and prohibiting certain pages will, therefore, not solve the problem of Web-based Jew-hatred. The most implicit manifestations encoded in indirect speech acts, would remain although they contain the most dangerous antisemitic content. The suppression of antisemitic contents online can therefore only be a first step and has to be accompanied by the same positive counter-narratives that were recommended for cultural decision makers and influencers as well as by accurate and easily accessible information about the history of antisemitism and Jewish persecution, mentioned in the same chapter.

In line with our recommendations for cultural decision makers and influencers, a multifaceted approach is therefore recommended here that is characterized by repression and erasure of antisemitic contents, on the one hand, and by their replacement with positive representations of Judaism as well an accurate depiction of the history of antisemitism, on the other hand. Especially online, not the voice of the victimizers but the voice of the victims needs to be heard.

A special problem of combating antisemitism online is the limited role public institutions have played so far in this fight. NGOs were more active in monitoring and denouncing antisemitism online and in bringing this phenomenon to the attention of lawmakers and the general public. NGOs have also been more active than governments in flagging antisemitic content to social media and Internet providers and in helping other users to do so. These contents usually violate the terms of service, regardless if they are illegal in some countries. However, NGOs do not have the resources to monitor and flag antisemitic messages comprehensively. This is particularly true for less prominent languages. To change this situation, governments should become more active in monitoring online antisemitism, and Internet providers should offer user-friendly mechanisms and procedures for reporting hateful content while also enforcing their terms of service regarding the dissemination of hate speech.

A good example of how governments can support the fight against antisemitism online is the agreement on a "Community Code of Conduct" that 
European Commissioner Vera Jourova and Katharina von Schnurbein, the European Commission Coordinator on Combating Antisemitism, reached with the main Internet companies to identify antisemitic expressions and swiftly remove them. The implementation of this agreement is still far from complete and should be accelerated. Such co-operation between the Internet industry and governmental agencies might be the best way to further the fight against antisemitism online.

Because the Internet as a whole is part of the process of constructing cultural meaning, and because it is the latest repository of cultural and religious memories, many policies recommended for cultural decision makers and influencers apply to Internet decision makers and influencers as well. Given that the Internet provides antisemites with new, unparalleled technology to spread Jew-hatred and to poison minds, the following specialized recommendations are suggested that aim both at the short-term suppression and at the long-term eradication of antisemitism. Next to monitoring antisemitism online, they focus on its suppression and replacement by positive contents about Judaism.

\section{Monitoring Antisemitism Online}

- On the Internet, strategies to combat antisemitism have to focus on "everyday" users and not only on the Web pages of right wing extremists because these "everyday" promotors on the mainstream levels of the Web enhance the normalization and social acceptability of antisemitism. Monitoring of antisemitism online should thus not focus on right-wing extremists users only but also should address all forms of antisemitism including religious and left-wing Jew-hatred.

- The monitoring of antisemitism online is the responsibility of governments and should not be left to the activities of NGOs. Governments and international bodies should finance and encourage the finding of a solution to the monitoring of antisemitism on the Web. A good practice example is the Anti-Semitism Cyber Monitoring System of the Israeli Diaspora Ministry. Each country should have a similar system to monitor antisemitism online.

- In addition to government monitoring, social media and Internet providers need to take active monitoring measures. They also need to improve the supportive structure for flagging such content, take swift action when antisemitic messages are signaled to them, and provide more transparency for their efforts to combat cyberhate on their platforms.

- Online platforms should train their employees to recognize and eradicate the all forms of antisemitism as well as antisemitic code words. 
- Important approaches to monitor antisemitism on the Web include expert solicitation, automation through artificial intelligence, and crowd sourcing.

- A further key to successfully monitor and flag antisemitism online is intensified international cooperation between different public institutions, NGOs, lawmakers, and IT.

- All monitoring of antisemitism online has to be public and thus publicly accountable.

- The results of all monitoring of antisemitism online should be provided to state and federal agencies for criminal investigation, if appropriate. Otherwise, they should be communicated to the Internet industry for the removal of antisemitic contents from their platforms.

\section{Suppressing Antisemitism Online}

- Those who post antisemitic contents online always need to be held accountable. History proves that antisemitic agitation leads to the most violent crimes. Anyone who is involved in antisemitic agitation online should thus be prosecuted by the legal agencies.

- Once identified, all antisemitic Webpages, blogs, wikis, platforms, etc. need to be removed.

- Social bots, fake accounts, etc. through which antisemitic hate speech is spread should be deleted.

- Internet search engines can help to suppress antisemitism by excluding all antisemitic contents from their search.

- Left-wing platforms frequently include pro forma denunciations of racism, hate speech, and antisemitism but nonetheless engage in anti-Zionist propaganda, often with antisemitic connotations. As a consequence, extremist political anti-Israel discourse goes unchallenged as hate speech. This discourse should also be banned online.

- Many influential social media personalities disseminate antisemitic posts regularly, often using the exact same messages, with broad reach that garners attention via likes, comments and reposts. On the other hand, large numbers of antisemitic posts (shared by the general public) garner low interest and a low level of virality online. Closing influential antisemitic accounts and/or taking such posts and all correlating re-posts offline can significantly reduce the volume of online antisemitic content.

- Intensified international cooperation between different public institutions, NGOs, lawmakers, and IT can help to take antisemitic content offline, prevent its republishing under different names and so the migration of hateful 
content to unregulated platforms. Expressions of antisemitism that are identified on the Internet at local levels must be confronted globally in order to prevent their reappearance on servers in other countries.

- All online platforms should be made liable for any kind of hate speech that is expressed on them. In their function in the virtual world, they are equivalent to a publishing house, journal, or TV channel. Hence, they are responsible for the hate that is spread through them.

- Until removed, antisemitic contents of the Internet need to be accompanied by counter-narratives that identify them as antisemitic and emphasize the viewpoint of the victims.

- Governments, NGOs and the general public at large should increase pressure on platform operators to combat antisemitism online.

- Each country should develop special legislation against cyber-antisemitism and antisemitic contents on the Internet, if it has not done so already (see recommendations regarding legislatures).

- The Internet platforms of countries that are predominantly antisemitic need to be monitored by the international community of states. Their antisemitic agitation should be blocked from the Internet of all democratic countries.

\section{Replacing Antisemitism Online by Positive Depictions of Judaism and Accurate Depictions of the History of Antisemitism}

- Positive contents about Jewish history, culture and religion as well as modern Israel should be uploaded to the Web in high quality (see the recommendations for cultural decision makers). Existing contents of this nature should be highlighted.

- Accurate, high quality depictions of the history of antisemitism should be uploaded to the Web (see the recommendations for cultural decision makers). Existing contents of this nature should be highlighted.

- Both positive depictions of Judaism and high quality descriptions of the history of antisemitism need to be easily accessible and translated into all languages represented on the Web (see the recommendations for cultural decision makers).

- Internet search engines should privilege positive depictions of Judaism and accurate descriptions of the history of antisemitism. Advice on which contents to preference should be provided by the institutes for the critical 
study of antisemitism which we recommend be founded in each country (see recommendations for academic decision makers).

- Internet search engines and other online devices should highlight the voice of all victims of antisemitism (see below and recommendations for cultural decision makers).

- The voices of all victims of antisemitism from antiquity until today need be heard online in easily accessible online platforms translated into all languages present on the Web (a good practice example USC Shoah Foundation Institute for Visual History and Education [http://sfi.usc.edu/]).

- Wikis specializing in Jewish history, culture and religion should be created in all languages present on the Web. Existing wikis in English and Hebrew should be translated into all languages on the Web.

- A wiki dedicated to the modern State of Israel should be created in all languages present on the Web.

- A wiki specializing in a high quality depiction of the history of antisemitism should be created in all languages present on the Web.

- Free of charge online video games that familiarize the users with Judaism and provide positive emotional experiences with Judaism, its history, culture, and religion should be created.

- Internet memes about Judaism could be an important tool for communicating positive contents about Judaism online to counter antisemitic agitation.

- Public influencers and celebrities should help to counter hate speech on the Internet.

\section{Further Research}

Further research about antisemitism in the virtual world is needed and should be actively solicited by public institutions. Examples for topics of such research include:

- Automated artificial intelligence tools to monitor and erase antisemitic contents.

- How to create an early warning system online.

- How to develop effective counter-speech that responds to-and opposesantisemitic defamation. Current strategies of counter-narratives are too labor-intensive to be implemented on a larger scale. Semiautomated messages that are tailored for certain profiles or messages might help to improve reach and effectiveness.

- Combating antisemitic memes.

- The spread of antisemitism in on- and offline videogames. 


\section{To Summarize}

As the main transmitter of Jew-hatred, the Internet is responsible for the explosion of antisemitism in the last decades. A multifaceted approach is therefore recommended here that is characterized by repression and erasure of antisemitic contents, on the one hand, and by their replacement with positive representations of Judaism as well an accurate depiction of the history of antisemitism, on the other hand. Especially online, not the voice of the victimizers but the voice of the victims needs to be heard. 